\title{
Del abandono educativo temprano al reenganche formativo: un estudio narrativo con biogramas ${ }^{1}$
}

\section{From Earlier School Leaving to Educational Re- engagement: a Narrative Study with Biograms}

\author{
José Miguel Nieto Cano² \\ nietos@um.es \\ ANTONIO PORTELA PRUAÑO \\ aportela@um.es \\ Ana TORres Soto \\ ana.t.s@um.es \\ María Jesús Rodríguez Entrena \\ entrena@um.es \\ Universidad de Murcia, España
}

\section{Resumen:}

El estudio explora los motivos que Ilevaron en el pasado a los alumnos ${ }^{3}$ a una trayectoria que culminó en el abandono escolar temprano. Partiendo de este objetivo general, la investigación realizada tiene carácter exploratorio y heurístico, adoptando una metodología cualitativa conducente a obtener conocimiento ligado a la experiencia, significado y contexto de los sujetos protagonistas. Para ello, y

\begin{abstract}
:
The study explores the motives that, according to the students themselves, have led them in the past to a trajectory that culminated in early school leaving. Based on this general objective, the study carried out has an exploratory and heuristic nature and adopted a qualitative methodology in order to obtain knowledge linked to the experience, meaning and context of the protagonist subjects. To do this, and
\end{abstract}

1 Este trabajo se enmarca en el proyecto de investigación "La reincorporación formativa en la Escuela de la Construcción de Ceuta desde la perspectiva de sus estudiantes: un estudio de caso para la identificación de buenas prácticas", seleccionado por el Instituto de Estudios Ceutíes en la Convocatoria de Ayudas a la Investigación correspondiente a 2015. A ambas entidades expresamos nuestro profundo agradecimiento.

2 Dirección para correspondencia (correspondence address):

José Miguel Nieto Cano. Dpto. de Didáctica y Organización Escolar. Universidad de Murcia. Facultad de Educación. Campus de Espinardo, s/n. 30100 Murcia (España).

3 A lo largo de este trabajo, y salvo que se indique lo contrario, utilizamos el plural masculino con valor genérico para hacer referencia a sujetos participantes de ambos géneros. 
en el marco de un estudio de caso, se ha recurrido a entrevistas semiestructuradas en profundidad con el alumnado joven perteneciente a la Escuela de la Construcción en Ceuta, que oferta itinerarios formativos para la inserción socio-laboral de personas en situación o riesgo de exclusión social. El procedimiento ha permitido recoger información sobre la trayectoria de los alumnos hasta acceder al centro. Los relatos han sido sometidos a un análisis narrativo, adoptando los resultados el formato de biogramas con categorías referidas a periodos vitales que permitieran dar cabida a singularidades asociadas a cada situación personal. Los resultados obtenidos indican que la reanudación de la trayectoria formativa no sólo depende de la propia formación sino también de la trayectoria previa de los sujetos. Existen razones que, desde los centros, empujan a los alumnos a abandonar su educación $y$, desde fuera de ellos, a sacarles de la educación, teniendo asimismo ambos tipos de razones incidencia en la posterior decisión de reanudar su formación.

\section{Palabras clave:}

Abandono educativo; fracaso escolar; jóvenes desfavorecidos; estudiantes en riesgo; renganche formativo; análisis de trayectorias; historias de vida. within the framework of a single case study, we have used semi-structured interviews in depth with the young students belonging to the Escuela de la Construcción in Ceuta, which offers training itineraries for the socio-labor insertion of people in situation or risk of social exclusion. This procedure has allowed us to collect information about the trajectory of the students until they were admitted to the center. The stories were subjected to narrative analysis and the results adopted the biogram format, using categories referring to vital stages and scope that allow for the accommodation of the singularities associated with each personal situation. The findings suggest that the resumption of the formative trajectory depends not only on training itself but also on the previous trajectory of the subjects. Reasons pushing students to abandon their education come both from the education centers and from outside them and that both types of reasons will also have an impact on the subsequent decision to resume their training.

\section{Key words:}

Educational dropout; educational failure; disadvantaged youth; at risk students; educational re-engagement; path analysis; life stories.

\section{Résumé :}

L'étude approfondit les causes qui ont porté aux apprenants, dans le passé, à un parcours éducatif qui a abouti à l'abandon scolaire précoce. En fonction de cet objectif général, l'étude, réalisée d’un point de vue exploratoire et heuristique, a adopté une méthodologie qualitative afin d'obtenir connaissance indissociable de l'expérience, de la signification/importance et du contexte des sujets protagonistes. Pour y réussir, et dans le cadre d'une conception de cas unique, on a eu recours à des entretiens semi-structurées et détaillées aux étudiants appartenant à l'Éscuela de la Construcción de Ceuta, laquelle offre des programmes de formation pour l'insertion socioprofessionnelle aux personnes en situation ou en risque d'exclusion sociale. Le procédé a permis de recueiIlir d’information sur le parcours de vie des élèves jusqu'à leur arrivée au centre éducatif. Les récits autobiographiques ont été soumis à un analyse narratif après lequel les résultats ont adopté la forme des biogrammes y compris des catégories relatives aux périodes de vie permettant de tenir compte des singularités liées à chaque situation personnelle. Les résultats obtenus montrent que la reprise de la trajectoire formative dépend non uniquement de la formation elle-même mais aussi du parcours de vie des individus. Il existe des raisons qui, dès l'intérieur de l'institution formatrice, poussent aux élèves à l'abandon de leur formation au même temps que, en dehors d'elle, il y a d'autres qui les incitent à quitter leur processus éducatif. Ces deux types de raisons auront par ailleurs incidence sur l'ultérieure décision de recommencer leur formation scolaire. 
José Miguel Nieto Cano, Antonio portela pruaño, Ana Torres Soto y María Jesús Rodríguez

\section{Mots clés:}

Abandon scolaire; échec scolaire; jeunes défavorisés; étudiantes à risque; reprise formative; analyse des trajectoires; histoires de vie.

Fecha de recepción: 7-2-2018

Fecha de aceptación: 15-5-2018

\section{Introducción}

El desempleo entre la población joven es un fenómeno ampliamente extendido, agudizado por la crisis económica. Si bien ello no significa que se distribuya por igual en todos los países e incluso dentro de un mismo país, sí está considerado que a escala global estaría en aumento, siendo generalizada la preocupación que suscita (International Labour Office, 2016). Por lo demás, ligado al desempleo de la población en general, los jóvenes serían más vulnerables al desempleo y los efectos de este pueden resultar más acusados y prolongados (Scarpetta, Sonnet y Manfredi, 2010; O’ Higgins, 2012). La condición de desempleado juvenil y la de joven que no estudia ni trabaja han sido asociadas al abandono escolar temprano y al escaso nivel formativo ligado al mismo. La incidencia de una y otra condición es mayor entre quienes abandonan tempranamente la educación escolar (OECD, 2014). Este último fenómeno ha sido considerado como proceso prolongado, que pudiendo iniciarse en fases tempranas, finaliza con la salida de la escuela (Rumberger, 2011), en ocasiones, dirigiendo a los alumnos hacia empleos poco cualificados (Yates, Harris, Sabates y Staff, 2011).

En este contexto, el impacto negativo ejercido por el abandono educativo temprano en el desempleo e inactividad juvenil (más las situaciones de exclusión social que estos pueden acarrear) pone de relieve la importancia de la educación y la formación en ese ámbito (Woessman, 2016). Ambas pueden ser responsabilizadas de ser, al menos parcialmente, tanto causantes de estos problemas como remedio a los mismos, por la importante contribución que pueden hacer para afrontarlos con éxito (Hoeckel, 2014). Así, en un entorno socioeconómico donde la transición entre el ámbito de la educación y la formación y el ámbito del empleo es más difusa, compleja y prolongada y donde el aprendizaje a lo largo de la vida se hace cada vez más necesario, resultan crecientemente importantes y necesarias las iniciativas conducentes a tomar medidas "compensatorias" y crear oportunidades de reanudar la formación (post- 
José Miguel Nieto Cano, Antonio portela pruaño, Ana Torres Soto y María Jesús Rodríguez ENTRENA

obligatoria, profesional, ocupacional) dirigidas a personas desempleadas en riesgo de exclusión social (Eichhorst y Rinne, 2016; O`Higgins, 2017). Por lo demás, el abandono educativo temprano estaría entre los retos principales a los que ha de hacer frente el sistema educativo español (Tarabini, 2015). Los propios expertos e instituciones europeas reconocen que los fenómenos del abandono escolar temprano, del desempleo y de la inactividad entre los jóvenes, son complejos y pueden ser atribuidos a un entramado de factores numerosos y heterogéneos, en los que no siempre resulta fácil, ni simple, introducir cambios significativos.

Con todo, las deficiencias en la educación y la formación adquiridas son decisivas al respecto. También lo es el desapego de los alumnos hacia la educación escolar, que a menudo es atribuido a la falta de motivación o incluso es asimilado a ésta. En esta falta de implicación o desapego intervienen una constelación de condicionantes en interacción, entre los cuales hay condicionantes personales y sociales, pero también condicionantes educativos y escolares que pueden afectar al impacto de los primeros (Fredricks, 2014). De este conjunto de circunstancias se deriva el interés de explorar las razones, motivos y otros condicionantes que, desde la perspectiva de alumnos con trayectoria previa de fracaso y abandono escolar temprano, les han inducido en el pasado a interrumpir su proceso educativo y les inducen en el presente a decidir reincorporarse a su formación en un centro e implicarse en ella. El trabajo que aquí se presenta presta una atención especial a los motivos para abandonar, si bien teniendo presente la relación (de asimilación más que de diferenciación) que se establece entre éstos y los motivos para "re-engancharse" como alternativa de solución a su situación personal.

Son centro de interés, pues, los posibles factores o causas que determinan tanto la situación de abandono temprano de la educación como la situación de eventual reanudación de la trayectoria educativa tras producirse aquella, asumiendo que ambas situaciones se constituyen como el resultado de una elección o decisión (Blenkinsop, McCrone, Wade y Morris, 2006; Higgins, Vaughan, Phillips y Dalziel, 2008). En estas coordenadas teóricas, el objetivo del estudio es explorar las razones, acontecimientos y circunstancias que a los alumnos llevaron en el pasado a una trayectoria educativa que culminó con el abandono escolar temprano y que, desde su punto de vista, son relevantes para explicar el proceso que en el presente ha culminado en su re-enganche, dando ahora continuidad a esa trayectoria en un centro formativo. 


\section{Marco empírico}

\section{Método}

Para obtener evidencia relevante que permita responder, con el menor grado de incertidumbre posible, al objetivo planteado, el diseño de la investigación adopta la estructura y la estrategia de un estudio de caso exploratorio e incluso heurístico de naturaleza cualitativa y emergente.

Por una parte, la metodología es de carácter cualitativo en el sentido básico de que los datos recogidos y analizados tienen un carácter cualitativo, al ser considerados estos los más adecuados para aprehender y comprender las experiencias de unas personas en un entorno particular (precisamente, el entorno natural donde es llevada a cabo la investigación), accediendo al sentido o significado que allí confieren a esas experiencias (Merriam y Tisdell, 2016). Por otra parte, la metodología es de carácter emergente, lo que significa que, precisamente en la búsqueda de información relevante por parte de los participantes en un escenario 'natural' sobre el que no es posible ejercer el control, el plan de trabajo ha de ser suficientemente flexible, dinámico y evolutivo, incorporando cambios para adecuarse al conocimiento que se va adquiriendo en el curso del proceso investigador y a la situación en que este está desarrollándose (Creswell, 2013; Morgan, 2008). Esta metodología ha sido particularmente relevante en la realización de este estudio, debido a su pertinencia para generar conjeturas y proposiciones que puedan ser desarrolladas posteriormente con mayor alcance, profundidad y detalle (George y Bennett, 2005).

Dado que este estudio tiene un propósito heurístico es singularmente relevante la selección de un caso con interés instrumental, dado que mediante el estudio pormenorizado del mismo se consigue la comprensión de algo que va más allá del mismo (Thomas y Myers, 2015). De acuerdo con la tipología propuesta por Yin (2014), esta investigación ha seleccionado un caso único con múltiples unidades de análisis o integrado.

\section{Muestra}

Atendiendo al objetivo del estudio, el caso seleccionado es la Escuela de la Construcción de la Ciudad Autónoma de Ceuta y es adoptado como caso único al revestir una singularidad que lo hace difícilmente com- 
parable a otros centros en relación tanto al entorno local como a otros entornos. Entre sus cometidos principales están las actuaciones dirigidas a la inserción y reinserción en la sociedad de personas desempleadas en riesgo de exclusión social, mediante la formación e inserción laboral. Las unidades de análisis están centradas en los alumnos jóvenes del centro según hayan estado o no en situación de abandono educativo temprano antes de acceder al centro y las perspectivas diferenciadas que puedan ser consideradas.

Así, la información presentada está referida a 39 alumnos vinculados a la Escuela de la Construcción en el curso 2015-16 que aceptaron voluntariamente la invitación a participar en la investigación. Todos son jóvenes cuyas edades oscilan entre los 18 y los 24 años. Atendiendo a sus declaraciones en torno a su trayectoria educativa y formativa previa a su incorporación al centro, hemos agrupado los casos individuales de los que va a ser presentada la información en función de presentar o no la condición de abandono educativo temprano (en adelante, alumnos AET y alumnos NO AET, respectivamente).

El indicador utilizado por el Instituto Nacional de Estadística en su encuesta de población activa (EPA), refiere el abandono temprano de la educación y la formación como "las personas que tienen entre 18 y 24 años, que han abandonado sus estudios al finalizar la etapa obligatoria o con anterioridad y que no han realizado ningún curso de formación reglada o no reglada durante las cuatro semanas antes inmediatamente anteriores a la encuesta" (INEE, 2014). Sin embargo, debido a circunstancias emergentes que afectaban a la planificación y desarrollo del trabajo de campo nuestra conceptuación del AET no contempla expresamente la segunda de las condiciones señaladas en la definición, de manera que algunos participantes estaban compaginando el curso de la Escuela de la Construcción con otras acciones de educación post-obligatoria dentro del sistema educativo o desligadas de éste (véase Tabla 1). 
Del abandono educativo temprano al reenganche formativo: un estudio narrativo con biogramas

José Miguel Nieto Cano, Antonio portela pruaño, Ana Torres Soto y María Jesús Rodríguez

Tabla 1. Participantes en el estudio de caso.

\begin{tabular}{|c|c|c|c|}
\hline Condición de alumnos jóvenes & Edad & Participantes & Totales \\
\hline \multirow{8}{*}{ AET } & 24 & $\mathrm{~J} 1, \mathrm{~J} 2$ & 2 \\
\hline & 23 & $\mathrm{~J} 3, \mathrm{~J} 4, \mathrm{~J} 5, \mathrm{~J} 6$ & 4 \\
\hline & 22 & $\mathrm{~J} 7 * *, \mathrm{~J} 8, \mathrm{~J} 9 * * ; \mathrm{J} 10^{* *}, \mathrm{~J} 11^{*}$ & 5 \\
\hline & 21 & $\begin{array}{l}\mathrm{J} 12, \mathrm{~J} 13, \mathrm{~J} 14, \mathrm{C} 15^{* * *}, \mathrm{~J} 16, \\
\mathrm{~J} 17, \mathrm{~J} 18\end{array}$ & 7 \\
\hline & 20 & $\mathrm{~J} 19 *, \mathrm{~J} 20, \mathrm{~J} 21$ & 3 \\
\hline & 19 & $\mathrm{~J} 22, \mathrm{~J} 23, \mathrm{~J} 24, \mathrm{~J} 25$ & 4 \\
\hline & 18 & $\mathrm{~J} 26, \mathrm{~J} 27, \mathrm{~J} 28, \mathrm{~J} 29$ & 4 \\
\hline & \multicolumn{2}{|c|}{ TOTAL AET } & 29 \\
\hline \multirow{8}{*}{ NO AET } & 24 & $\mathrm{~J} 30, \mathrm{~J} 31, \mathrm{~J} 32, \mathrm{~J} 33$ & 4 \\
\hline & 23 & $\mathrm{~J} 34$ & 1 \\
\hline & 22 & $\mathrm{~J} 35, \mathrm{~J} 36$ & 2 \\
\hline & 21 & $\mathrm{~J} 37$ & 1 \\
\hline & 20 & $\mathrm{~J} 38, \mathrm{~J} 39$ & 2 \\
\hline & 19 & - & 0 \\
\hline & 18 & - & 0 \\
\hline & TOTA & L NO AET & 10 \\
\hline TOTAL & & & 39 \\
\hline
\end{tabular}

Fuente: Elaboración propia.

(*) Compaginaría estudios de Formación Profesional de Grado Medio.

$\left.{ }^{* *}\right)$ Compaginaría estudios de Bachillerato.

${ }^{(* *)}$ Compaginaría curso del INEM (Instituto Nacional de Empleo).

\section{Instrumentos}

El estudio indaga en la perspectiva que tienen los alumnos, fundamentándose en la evidencia verbal, con el propósito de introducir las menos restricciones posibles en la expresión de los participantes y, a la vez, ampliar las oportunidades de expresión en la máxima medida. En congruencia, la recogida de datos se llevó a cabo mediante entrevistas individuales en profundidad. Asimismo, en consonancia con el objetivo y el diseño de la investigación, las entrevistas tuvieron un carácter semiestructurado por lo que se desarrollaron en torno a un conjunto de cuestiones básicas referidas a tópicos delimitados y secuenciados con antelación (en forma de guía de entrevista) por el equipo investigador (y anticipados previamente a cada informante según un protocolo de en- 
José Miguel Nieto Cano, Antonio portela pruaño, Ana Torres Soto y María Jesús Rodríguez ENTRENA

trevista preparado al efecto), si bien otra parte de las preguntas concretas fueron formuladas en el curso de la entrevista, según esta avanzaba, atendiendo a las respuestas proporcionadas por los mismos informantes (Patton, 2015; Rubin y Rubin, 2012).

Tomando como referencia y adaptando las propuestas por Mills, McGregor y te Riele (2015, p. 156) y por Smyth y Robinson (2015, p. 223), la pregunta básica en la guía de entrevista referida al objeto de este trabajo era ¿cuál ha sido tu historia hasta venir a este centro?, y se asociaba a esta un conjunto de preguntas más específicas a las que poder recurrir para obtener una respuesta lo más completa y precisa posible a la misma:

- ¿cómo ha sido que vinieras al centro [la Escuela de la Construcción]?;

- ¿en qué otros centros has estado?, ¿qué destacarías, tanto positivo como negativo, de tu experiencia en ellos?;

- en todo este tiempo que ha pasado hasta llegar aquí, ¿qué destacarías de tu vida en general, sea positivo como negativo?;

- de todo lo que te ha venido ocurriendo, ¿qué es lo que más ha influido para que vinieras a este centro?

Posteriormente a la realización de las treinta y nueve entrevistas individuales en profundidad en un primer ciclo, y tomando como referencia el análisis preliminar de los datos, se realizaron en un segundo ciclo veintiuna entrevistas individuales y selectivas de confirmación entre los informantes del grupo de alumnos indicado (Cresswell, 2013). Todas las entrevistas fueron grabadas, con el consentimiento por escrito de los participantes. Asimismo, fueron transcritas íntegramente las correspondientes al primer ciclo y, por su carácter corroboratorio, fueron transcritas parcialmente las correspondientes al segundo ciclo.

\section{Análisis de la información}

Se recurrió específicamente al análisis narrativo para indagar en la narrativa que ofreció cada alumno sobre su trayectoria hasta acceder al centro (excluyendo los detalles relativos al proceso de acceso a este). La utilización de este enfoque para analizar esta información estuvo justificada por el formato narrativo en que tendió a ser presentada por parte de los alumnos y por la naturaleza idiosincrásica y personal de aquello a lo 
que estaba referida, al punto de que contribuía a presentar la identidad de cada uno (Creswell, 2013). En consecuencia, la codificación de esa información recurrió a categorías amplias referidas a etapas y ámbitos vitales, que permitieran dar cabida a información referida a tantas singularidades asociadas a cada situación personal.

Para el análisis de esta información se recurrió a biogramas, habitualmente aplicados al análisis de experiencias que, siguiendo un eje temporal, han marcado su trayectoria vital o, en su caso, motivado otros desarrollos posteriores y "cambios de rumbo" en la vida de una persona (Bolívar y Gijón, 2008). También han sido utilizados en investigaciones con alumnos en riesgo de exclusión social y educativa en nuestro país (Hernández de la Torre, 2013; Portela, Nieto y Toro, 2009; Rojas, Susinos y Calvo, 2013). Esta técnica consiste en construir una representación textual y visual sumaria de los sucesos más relevantes acaecidos en la vida de una persona, combinados y ordenados cronológicamente, en la cual quedan plasmados la descripción que hace la persona de los sucesos en sí y las apreciaciones o valoraciones de que son objeto por parte de ella, incluyendo las repercusiones que les son atribuidas y los contextos (personales, familiares, económico, laborales) en que ocurren, otorgándoles sentido (Suárez-Ortega, 2012). Al tener un carácter sumario, a diferencia de los relatos y las historias de vida, pueden presentarse como registros biográficos de un amplio número de biografías personales (Martín García, 1995) lo que facilita realizar comparaciones, así como sugerir, ilustrar o contrastar hipótesis o conjeturas aplicables al conjunto de las mismas, pese a la singularidad de la trayectoria de cada participante. Finalmente, también se ha recurrido a prácticas próximas al análisis de contenido, ya que, una vez disponible el sistema final de códigos y categorías, ha sido determinada a través de frecuencias la distribución de los mismos (Silverman, 2014), con la ayuda del programa de análisis de datos ATLAS.ti (versión 7).

\section{Resultados y discusión}

Los resultados del análisis se ordenan en dos partes. La primera abarca el periodo de educación obligatoria, dando cuenta particularmente de las experiencias vividas durante la etapa de Educación Secundaria Obligatoria. La segunda corresponde al periodo posterior hasta el momento 
José Miguel Nieto Cano, Antonio portela pruaño, Ana Torres Soto y María Jesús Rodríguez ENTRENA

de acceder a la Escuela de la Construcción. Los datos son presentados a través de una descripción narrativa acompañada de citas literales ilustrativas, provenientes de las entrevistas realizadas y de los biogramas elaborados a partir de cada una de ellas.

En cuanto al periodo de la educación obligatoria, dentro del conjunto de alumnos AET (veintinueve alumnos), trece de ellos la completaron, obteniendo el título de Graduado en Educación Secundaria Obligatoria, pero son más (dieciséis alumnos) los que no completaron en su momento esta etapa y, en consecuencia, no estaban, en el momento de acceder a la Escuela de la Construcción, en posesión del título oficial correspondiente ni, por supuesto, de ningún otro más avanzado dentro del sistema educativo. Pero, asimismo, algunos de los alumnos NO AET ponen también de relieve al ser entrevistados situaciones similares a las planteadas por aquellos, por lo que también se hará referencia a ellas ocasionalmente.

En general, los alumnos no señalan situaciones destacables acaecidas durante el período de Educación Primaria (o anterior); . prácticamente en todos los casos, los problemas comienzan a producirse durante su tránsito a la Educación Secundaria Obligatoria (en lo sucesivo, ESO) y se manifiestan en los suspensos y la repetición de cursos. En algunos de los casos (once de los dieciséis que no titularon en esta etapa), la repetición de curso, sin éxito alguno, también precedió el abandono los estudios de Educación Secundaria Obligatoria y, por ende, el sistema educativo:

En el instituto dejé los estudios; no terminé la ESO; lo dejé en cuarto. (J1) No terminé la ESO; en el segundo trimestre me fui. (J3)

En otros casos, la repetición de cursos precederá al acceso a un Programa de Cualificación Profesional Inicial (en lo sucesivo, PCPI), como medida de segunda oportunidad a la que no todos tendrán acceso. No obstante, tampoco esta medida beneficiará a todos por igual. De los veintinueve alumnos AET entrevistados, diez manifestaron haber cursado un $\mathrm{PCPI}$, pero únicamente seis (de los trece casos AET que titularían en esta etapa) habrían logrado revertir la situación, completando finalmente con éxito esta etapa y obteniendo el correspondiente título. Un caso de los alumnos NO AET, también tituló tras abandonar el instituto y realizar varios $\mathrm{PCPI}$, el resto no lo lograría.

Las declaraciones de los alumnos entrevistados nos pueden ayudar a 
comprender con mayor claridad qué circunstancias del contexto están en la base de situaciones problemáticas o negativas, las cuales, en la mayoría de los casos no son únicas y comunes para todos, sino múltiples y diversas (véase Tabla 2). Así, nos encontramos a veces con explicaciones que hacen referencia a cambios acaecidos en su situación en ese periodo de su trayectoria (los cuales ocurren también puntualmente en casos de alumnos NO AET), frente a los cuales cabría colocar su falta de adaptación o ajuste a los mismos con una respuesta suficientemente satisfactoria:

Tabla 2. Circunstancias externas aducidas por los alumnos.

\begin{tabular}{|c|c|c|c|}
\hline $\begin{array}{l}\text { Factores } \\
\text { aducidos }\end{array}$ & $\begin{array}{l}\text { AET } \\
\text { Frecuencia }\end{array}$ & $\begin{array}{l}\text { NO AET } \\
\text { Frecuencia }\end{array}$ & Ejemplos \\
\hline \multirow[t]{3}{*}{$\begin{array}{l}\text { Familia de } \\
\text { referencia }\end{array}$} & $10 / 29$ & $1 / 10$ & $\begin{array}{l}\text { Porque mi padre estaba en la cárcel... } \\
\text { y luego, lo único que hacía era pasar de } \\
\text { mí y yo ver cosas malas nada más, no } \\
\text { podía seguir. (J6) }\end{array}$ \\
\hline & & & $\begin{array}{l}\text { Porque mi madre se puso mala. Y estu- } \\
\text { ve con ella fuera con lo de la quimiote- } \\
\text { rapia. (J25) }\end{array}$ \\
\hline & & & $\begin{array}{l}\text { Faltaba a clase por buscar trabajo por- } \\
\text { que en casa siempre había problemas } \\
\text { económicos. (J35) }\end{array}$ \\
\hline \multirow[t]{2}{*}{$\begin{array}{l}\text { Paternidad/1 } \\
\text { maternidad }\end{array}$} & $3 / 29$ & $1 / 10$ & $\begin{array}{l}\text { Me quedé embarazada. Aquello se me } \\
\text { puso cuesta arriba y lo dejé, ya está. (J3) }\end{array}$ \\
\hline & & & $\begin{array}{l}\text { Mi niño tiene ya } 4 \text { años. Y estoy con } \\
\text { mi novia, ella también tiene un niño. } \\
\text { Cuando ella está trabajando me los } \\
\text { quedo yo, y no puedo sacarme la ESO } \\
\text { por las tardes porque ahora mismo ella } \\
\text { está trabajando. (J4) }\end{array}$ \\
\hline
\end{tabular}


Del abandono educativo temprano al reenganche formativo: un estudio narrativo con biogramas

José Miguel Nieto Cano, Antonio portela pruaño, Ana Torres Soto y María Jesús Rodríguez ENTRENA

\begin{tabular}{|c|c|c|c|}
\hline $\begin{array}{l}\text { Factores } \\
\text { aducidos } \\
\end{array}$ & $\begin{array}{l}\text { AET } \\
\text { Frecuencia }\end{array}$ & $\begin{array}{l}\text { NO AET } \\
\text { Frecuencia }\end{array}$ & Ejemplos \\
\hline \multirow[t]{2}{*}{$\begin{array}{l}\text { Entorno } \\
\text { social y } \\
\text { grupo de } \\
\text { compañeros } \\
\text { en clase }\end{array}$} & $9 / 29$ & $1 / 10$ & $\begin{array}{l}\text { Cuando ya pasamos al edificio grande, } \\
\text { ya hay gente más mayor, hay malas in- } \\
\text { fluencias y tal, y eso es lo que me pasó. } \\
\text { Me junté con malas personas. Menos } \\
\text { mal que no caí en droga ni nada de eso. } \\
\text { (J6) }\end{array}$ \\
\hline & & & $\begin{array}{l}\text { En el instituto al ser más chico no sa- } \\
\text { bía lo que hacía, te daba igual todo... Y } \\
\text { después he desperdiciado eso por irme } \\
\text { con los compañeros... Y también, por } \\
\text { el barrio en el que vivo, El Príncipe, y } \\
\text { allí se ven cosas. (J22) }\end{array}$ \\
\hline \multirow[t]{2}{*}{$\begin{array}{l}\text { Centro } \\
\text { educativo, } \\
\text { profesores y } \\
\text { enseñanza }\end{array}$} & $8 / 29$ & $1 / 10$ & $\begin{array}{l}\text { Pero en el instituto, entraba un montón } \\
\text { de gente y los profesores allí explican- } \\
\text { do y explicando y nosotras allí. (J1) } \\
\text { Había más teoría y eso no interesa. (J14) }\end{array}$ \\
\hline & & & Los profesores pasaban. (J27) \\
\hline
\end{tabular}

Fuente: Elaboración propia.

En efecto, a menudo lo que los alumnos AET (y, puntualmente, algunos alumnos NO AET) aducen como explicaciones son cambios operados en sí mismos, que impiden o dificultan su adaptación a las nuevas situaciones que afrontaban en la educación obligatoria (véase, Tabla 3). En estos casos, pues, el patrón explicativo más concurrente consistiría en que aquello que motiva la experiencia problemática o negativa acaba estando, pues, en los propios alumnos: 
Del abandono educativo temprano al reenganche formativo: un estudio narrativo con biogramas

José Miguel Nieto Cano, Antonio portela pruaño, Ana Torres Soto y María Jesús Rodríguez

Tabla 3. Factores personales aducidos por los alumnos.

\begin{tabular}{llll}
\hline Factores & AET & NO AET & Ejemplos \\
aducidos & Frecuencia & Frecuencia & Yo también de joven era tonto..., estaba \\
\hline $\begin{array}{l}\text { Madurez } \\
\text { psicológica }\end{array}$ & $11 / 29$ & $3 / 10$ & $\begin{array}{l}\text { O tadido. (J5) } \\
\text { perdicion }\end{array}$
\end{tabular}

En el instituto, al ser más chico, no sabía lo que hacía, te daba igual todo. No seguí porque fui tonto, la verdad... Y ahora con 19 años ya te das cuenta de eso. (J22)

\begin{tabular}{|c|c|c|c|}
\hline & \multirow[t]{2}{*}{$\begin{array}{ll}\begin{array}{l}\text { Conducta } \\
\text { desajustada }\end{array} & \\
\end{array}$} & \multirow[t]{2}{*}{ 4/10 } & \multirow{2}{*}{$\begin{array}{l}\text { Muchas expulsiones..., en el reforma- } \\
\text { torio no llegué a reformarme al } 100 \% \\
\text { pero empecé asumir más metas, a qui- } \\
\text { tarme de las malas influencias y las ma- } \\
\text { las consecuencias que tenía antes. (J20) } \\
\text { Me iban a echar...la liaba mucho, se me } \\
\text { iba la pinza..., me tenían miedo y todo. } \\
\text { (J38) }\end{array}$} \\
\hline & & & \\
\hline \multicolumn{2}{|c|}{$\begin{array}{l}\text { Conductas } \quad 6 / 29 \\
\text { de riesgo }\end{array}$} & $0 / 10$ & $\begin{array}{l}\text { Cuando mi padre entró en la cárcel } m \\
\text { perdí, porque yo también entre con }\end{array}$ \\
\hline
\end{tabular}

Me metía de todo, con 15 ó 16 años. (J6)

Fuente: Elaboración propia.

La confluencia de varios factores, extrínsecos y/o intrínsecos, acabaría provocando una situación de desmotivación intensa y persistente en no pocos de los alumnos, lo que se reflejaría en un historial más o menos prolongado de desafección o inactividad, de expulsiones, de absentismo que finalizaría con el abandono de la ESO sin completarla. Un grupo de estos alumnos hacen explícitos en sus manifestaciones sentimientos de auto-inculpación y arrepentimiento por no haber sabido o sido capaces de aprovechar aquella oportunidad. Así lo explican algunos de ellos:

Y me siento decepcionado conmigo mismo, la única culpa se me tiene que echar a mí... Porque yo sé que si hubiera querido hubiera acabado mi ESO, mi Bachiller, hubiera llegado lejos si hubiera querido. (J18)

En aquellos momentos, el fallo era mío. (J24) 
Algunos de ellos, expresarían, asimismo, su deseo o su expectativa de restaurar en un futuro inmediato aquellos estudios que abandonaron en el pasado.

En el caso de los alumnos AET que finalmente titularon al final de la ESO, los episodios de abandono fueron transitorios. Los cambios en el contexto educativo y socio-familiar demandaron de los alumnos una reacción inmediata. Algo similar ocurre en el subgrupo de alumnos NO AET: los que experimentaron vivencias negativas y problemas (3 alumnos) supieron o fueron capaces de restaurarlas por ellos mismos y con el apoyo de familias o profesores.

En cuanto al subconjunto de alumnos jóvenes NO AET (diez alumnos), algunos de los entrevistados no hicieron referencia a la etapa de educación obligatoria. En términos comparativos y globales, es más frecuente en este subgrupo que los alumnos no destaquen problemas y les haya ido "bien". En relación con el periodo de la educación postobligatoria, su tránsito dentro del sistema educativo en términos de acreditación académica incluye los siguientes resultados en lo que se refiere a la titulación oficial más avanzada que ha sido alcanzada: (a) la mitad consiguió la titulación de Técnico (J31, J34, J35, J37, 38); (b) uno, la de Técnico Superior (J30); y (c) cuatro, lograron obtener la titulación de Bachiller (J32, J33, J36, J39).

En cuanto al subconjunto de alumnos AET, ninguno de los veintinueve casos, incluyendo los que obtuvieron el título de Graduado en ESO, Ilega a obtener un título más avanzado en el momento de acceder a la Escuela de la Construcción en el curso 2015-16 y realizar las entrevistas. Pero, ¿qué ocurrió después de la etapa de la educación obligatoria hasta el momento presente? ¿Qué experiencias educativas o formativas destacan los alumnos dentro del sistema educativo o desligadas del mismo? La Tabla 4 ofrece sintetizada la información declarada por los alumnos AET que fueron entrevistados. 
Del abandono educativo temprano al reenganche formativo: un estudio narrativo con biogramas

José Miguel Nieto Cano, Antonio portela pruaño, Ana Torres Soto y María Jesús Rodríguez

Tabla 4. Actividad realizada por alumnos AET durante la etapa post-obligatoria.

\begin{tabular}{|c|c|c|}
\hline Actividad & Frecuencia & $\begin{array}{l}\text { Alumnos } \\
\text { AET }\end{array}$ \\
\hline $\begin{array}{l}\text { Estar compaginando estudios para obtener } \\
\text { título de GESO (Educación de Adultos) }\end{array}$ & $4 / 29$ & $\mathrm{~J} 5, \mathrm{~J} 14, \mathrm{~J} 21, \mathrm{~J} 22$ \\
\hline $\begin{array}{l}\text { Estar compaginando estudios de FP de } \\
\text { Grado Medio sin concluir }\end{array}$ & $3 / 29$ & $J 11, J 16, J 19$ \\
\hline $\begin{array}{l}\text { Estar compaginando estudios de Bachille- } \\
\text { rato sin concluir }\end{array}$ & $3 / 29$ & $\mathrm{~J} 7, \mathrm{J9}, \mathrm{J} 10$ \\
\hline $\begin{array}{l}\text { Interrumpir obtención del título de GESO } \\
\text { (por acceso a Escuela de la Construcción) }\end{array}$ & $3 / 29$ & $\mathrm{~J} 4, \mathrm{~J} 18, \mathrm{~J} 21$ \\
\hline $\begin{array}{l}\text { Abandonar o interrumpir FP de Grado } \\
\text { Medio }\end{array}$ & $3 / 29$ & $\mathrm{~J} 11, \mathrm{~J} 15, \mathrm{~J} 25$ \\
\hline Abandonar o interrumpir Bachillerato & $3 / 29$ & $\mathrm{~J} 11, \mathrm{~J} 12, \mathrm{~J} 15$ \\
\hline $\begin{array}{l}\text { Haber realizado uno o varios cursos for- } \\
\text { mativos (en empresas, ocupacionales, etc.) }\end{array}$ & $10 / 29$ & $\begin{array}{l}\mathrm{J} 1, \mathrm{~J} 4, \mathrm{~J} 7, \mathrm{~J} 8, \mathrm{~J} 13, \mathrm{~J} 15, \\
\mathrm{~J} 17, \mathrm{~J} 22, \mathrm{~J} 123, \mathrm{~J} 25\end{array}$ \\
\hline Haber interrumpido o abandonado cursos & $2 / 29$ & $\mathrm{~J} 6, \mathrm{j} 17$ \\
\hline $\begin{array}{l}\text { Haber realizado curso del Polifuncional- } \\
\text { Equal como paso previo al acceso }\end{array}$ & $15 / 29$ & $\begin{array}{l}\mathrm{J} 1, \mathrm{~J} 3, \mathrm{~J} 8, \mathrm{J9}, \mathrm{J} 10, \mathrm{~J} 12, \\
\mathrm{~J} 15, \mathrm{~J} 17, \mathrm{~J} 18, \mathrm{~J} 19, \\
\mathrm{~J} 22, \mathrm{~J} 24, \mathrm{~J} 26, \mathrm{~J} 27, \\
\mathrm{~J} 29\end{array}$ \\
\hline $\begin{array}{l}\text { Haber realizado otros cursos en la Escuela } \\
\text { de la Construcción, completados o no }\end{array}$ & $3 / 29$ & $\mathrm{~J} 4, \mathrm{~J} 8, \mathrm{~J} 13$ \\
\hline $\begin{array}{l}\text { Estar compaginando en el presente curso } \\
\text { formativo (INEM...) }\end{array}$ & $1 / 29$ & $J 15$ \\
\hline Haber estado inactivo mucho tiempo & $7 / 29$ & $\begin{array}{l}J 6, J 13, J 14, J 18, J 20, \\
J 22, J 27\end{array}$ \\
\hline Haber buscado activamente empleo & $1 / 29$ & $\mathrm{~J} 3$ \\
\hline Haber trabajado esporádicamente & $4 / 29$ & $\mathrm{~J} 2, \mathrm{~J} 4, \mathrm{~J} 5, \mathrm{~J} 9$ \\
\hline Estar trabajando a tiempo parcial & $2 / 29$ & $\mathrm{~J} 5, \mathrm{~J} 7$ \\
\hline $\begin{array}{l}\text { Otras actividades no remuneradas (volun- } \\
\text { tariado) }\end{array}$ & $1 / 29$ & $\mathrm{~J} 10$ \\
\hline
\end{tabular}

Fuente: Elaboración propia.

Aunque tanto las trayectorias como las circunstancias individuales son diferentes en cada caso, pueden ser diferenciadas dos tipos de situaciones, entre las que a veces se producen solapamientos: por un lado, la que corresponde a los alumnos jóvenes que han mantenido una trayectoria educativa ligada al sistema educativo y, por otro, la que corresponde a quienes han optado por una trayectoria formativa desligada del mismo, centrada en reali- 
José Miguel Nieto Cano, Antonio portela pruaño, Ana Torres Soto y María Jesús Rodríguez ENTRENA

zar formación ocupacional o en empresas. Atendiendo a lo manifestado por los alumnos, menos frecuentes han sido bien la situación de empleo, aun cuando éste fuera temporal y esporádico, o bien la situación de inactividad muy prolongada hasta el acceso a la Escuela de la Construcción.

Entre quienes siguieron cursando enseñanzas comprendidas en la educación postobligatoria dentro del sistema educativo, están, naturalmente, aquellos que titularon al finalizar la ESO. En once de los trece casos que estaban en esa situación, se optó bien por continuar estudios bien en Bachillerato (seis alumnos), o bien en Formación Profesional de Grado Medio (cinco alumnos), aunque prácticamente la mitad de ellos los acabarían abandonando o interrumpiendo uno y/u otro (por desmotivación, por trabajo, por circunstancias familiares, etc.), mientras que el resto corresponde a los casos que en el momento presente están compaginando esos estudios con el curso en la Escuela de la Construcción:

Empecé a hacer el grado medio, lo aprobé y ahora estoy haciendo segundo estudiando aquí. Estoy haciéndolo a día de hoy a distancia. Me queda una asignatura que es la que intento aprobar y que me den el título. (19)

Para quienes buscaron participar en actividades educativas o formativas desarrolladas fuera del sistema educativo será común ir realizando a lo largo del tiempo varios y diversos cursos (lo que salga,... a lo que te Ilamen) ofertados por entidades del ámbito ocupacional (INEM, empresas) saliendo de uno e incorporándose a otro y alternando periodos transitorios de inactividad hasta acceder a la Escuela de la Construcción:

Porque si no tienes el graduado, por lo menos tener un buen currículum, estar bien formado, que tengas una buena titulación de qué es lo que sabes hacer. (J2)

La frecuencia más elevada corresponde a alumnos que declaran haberse inscrito o haber realizado el curso en el Centro EQUAL (quince alumnos) antes de acceder a la Escuela de la Construcción. Esta experiencia, por lo demás, suele ser muy bien valorada:

Al ir a ese curso tienes mucha ayuda, de psicólogos, de gente que te apoya, que te intenta buscar trabajo. Y te relacionas, conoces gente, estudias algo que no sabías. (J1) 
Del abandono educativo temprano al reenganche formativo: un estudio narrativo con biogramas José Miguel Nieto Cano, Antonio portela pruaño, Ana Torres Soto y María Jesús Rodríguez

Gracias al apoyo que recibí [en Centro Equal] descubrí que me gusta ayudar a la gente. Siempre estaba desanimado, siempre estaba escuchando que no podía... Hasta que entré ahí. (J10)

Aunque predomine en este periodo una actividad en la que se van sucediendo situaciones de estudio, formación y trabajo ocasional, alternándose con momentos de inactividad, no resulta raro que algunos alumnos (siete alumnos) destaquen haber pasado un tiempo relativamente prolongado de inactividad:

Salí de mi instituto sin nada, me quedé cuatro años sin hacer nada, sin estudios, sin nada. (J13)

Me tiré un año sabático, sin hacer nada. (J14)

Con relación a los alumnos NO AET entrevistados, podemos observar, en la Tabla 5, la información sintética acerca de sus experiencias declaradas en el tramo correspondiente a la educación post-obligatoria.

Tabla 5. Actividad realizada por alumnos NO AET durante la etapa post-obligatoria.

\begin{tabular}{|c|c|c|}
\hline Actividad & Frecuencia & $\begin{array}{l}\text { Alumnos } \\
\text { NO AET }\end{array}$ \\
\hline Completa el Bachillerato & $5 / 10$ & $\begin{array}{l}\mathrm{J} 30, \mathrm{~J} 32, \mathrm{~J} 33, \mathrm{~J} 36, \\
\mathrm{~J} 39\end{array}$ \\
\hline $\begin{array}{l}\text { Completa un ciclo formativo de FP de Grado } \\
\text { Medio }\end{array}$ & $4 / 10$ & $J 31, J 34, J 35, J 38$ \\
\hline $\begin{array}{l}\text { Completa dos ciclos formativos de FP de Gra- } \\
\text { do Medio }\end{array}$ & $1 / 10$ & J37 \\
\hline $\begin{array}{l}\text { Completa un ciclo formativo de FP de Grado } \\
\text { Superior }\end{array}$ & $1 / 10$ & $J 30$ \\
\hline $\begin{array}{l}\text { Abandona o interrumpe estudios de FPG Su- } \\
\text { perior por oferta de trabajo o por curso }\end{array}$ & $2 / 10$ & $J 31, J 36$ \\
\hline $\begin{array}{l}\text { Abandona o interrumpe estudios de FP de } \\
\text { Grado Medio }\end{array}$ & $1 / 10$ & $J 38$ \\
\hline $\begin{array}{l}\text { Haber realizado uno o varios cursos formati- } \\
\text { vos (INEM, Ayuntamiento, Sindicatos) }\end{array}$ & $2 / 10$ & $\mathrm{~J} 31, \mathrm{~J} 38$ \\
\hline $\begin{array}{l}\text { Haber preparado oposiciones a convocatorias } \\
\text { públicas sin éxito o con abandono }\end{array}$ & $2 / 10$ & $\mathrm{~J} 32, \mathrm{~J} 38$ \\
\hline $\begin{array}{l}\text { Haber realizado curso del Centro Equal como } \\
\text { paso previo al acceso }\end{array}$ & $1 / 10$ & J36 \\
\hline
\end{tabular}


Del abandono educativo temprano al reenganche formativo: un estudio narrativo con biogramas

José Miguel Nieto Cano, Antonio portela pruaño, Ana Torres Soto y María Jesús Rodríguez ENTRENA

\begin{tabular}{|c|c|c|}
\hline Actividad & Frecuencia & $\begin{array}{l}\text { Alumnos } \\
\text { NO AET }\end{array}$ \\
\hline $\begin{array}{l}\text { Estar compaginando en el presente otra activi- } \\
\text { dad educativa o formativa } \\
\text { (selectividad) }\end{array}$ & $1 / 10$ & J39 \\
\hline Haber estado inactivo (un año) & $2 / 10$ & $\mathrm{~J} 34, \mathrm{~J} 37$ \\
\hline $\begin{array}{l}\text { Haber buscado activamente empleo (INEM, } \\
\text { bolsas de trabajo, enviar currículum) }\end{array}$ & $2 / 10$ & $\mathrm{~J} 30, \mathrm{~J} 34$ \\
\hline
\end{tabular}

Fuente: Elaboración propia.

Como vemos, los estudios reglados de Bachillerato y de Formación Profesional de Grado Medio han sido los más cursados y, si bien el apoyo de la familia a seguir estudiando y evitar la inactividad está presente, los cursos formativos tendrán en un momento prioridad frente a los estudios reglados, aunque se mantenga la aspiración de retomarlos cuando las circunstancias (inactividad) lo hagan aconsejable.

\section{Conclusiones}

Este estudio ha perseguido indagar en las creencias de los alumnos jóvenes acerca de las causas que contribuyeron en el pasado a que abandonaran prematuramente la educación, considerando que aquellos aspectos a los que se refieren esas creencias pueden estar ejerciendo una influencia en la reanudación de la formación en el momento presente. Antes de abandonar prematuramente la educación, los alumnos estaban educándose en un centro escolar e identifican en esa situación aspectos que les proporcionaron razones para el abandono, empujándoles a ello. Asimismo, identifican en su situación general (familiar, económica, personal) aspectos que también les proporcionaron razones para abandonar su educación, contribuyendo así a sacarles de ella. Como señala Furlong (2013), el contexto social e institucional acaban obligando a elegir justificadamente los recorridos que realizan, a veces dando respuesta a serios problemas sociales que rebasan su alcance.

Y esta pauta es no ya sólo propia de quienes abandonaron prematuramente la educación sino también de quienes no lo hicieron. Implícitamente, pues, están haciéndose las siguientes conjeturas, para su contrastación a través del estudio: 
- Los alumnos tienen razones para actuar en un determinado sentido , i) bien favorable a mantener su participación e implicación en la actividad educativa/formativa en los centros y/o ii) a interrumpirla (lo que sería desfavorable para aquello).

- Tales razones son susceptibles de ser afectadas de forma relevante por la incidencia en la experiencia que tienen los alumnos de i) prácticas educativas/formativas u organizativas desde los centros y/o de ii) eventos y condicionantes desde fuera de los centros.

Pues bien, los resultados de nuestro estudio ponen de relieve que los factores internos de los centros que empujan a los alumnos a salir de ellos (push out) y los factores externos a los centros que sacan a los alumnos de los mismos (pull out), constituyen un amplio y heterogéneo grupo de aspectos al tiempo que ejercen una influencia que está sujeta a diferencias individuales (Caine, 2013; Campbell, 2015). En consecuencia, los patrones identificables varían, lo que corrobora que no habría pautas universales en las razones aducidas por los alumnos que abandonan la educación. Así, influyen en la conducta de abandonar prematuramente la educación desde intereses o necesidades que trascienden lo estrictamente educativo hasta cambios en la forma de pensar de los alumnos entrevistados a los que contribuyen personas cercanas con las que se relacionan.

En consonancia con la evidencia disponible, ello sería resultado de una elección o decisión por parte de los alumnos jóvenes, que estaría por igual determinando la conducta de reanudar eventualmente el proceso formativo, pues las decisiones, constituyan o no cambios significativos, tenderán a tener continuidad antes y después de las mismas, a través de múltiples elecciones contingentes que a veces llegan a ser efímeras sucediéndose impredeciblemente. Ello concuerda con la idea de la trayectoria vital en el sentido del recorrido que suelen seguir la mayoría de los jóvenes a través de numerosos puntos decisionales que ocasionalmente (no siempre) los encaminará en una dirección que suele ser, además, cambiante. Desde este punto de vista, los datos presentados sugieren que las razones que, desde los centros y desde fuera de ellos, llevan a los alumnos a abandonar su educación tienen equivalente incidencia en la posterior reanudación de la formación (Boylan y Renzulli, 2017). 
Del abandono educativo temprano al reenganche formativo: un estudio narrativo con biogramas

José Miguel Nieto Cano, Antonio portela pruaño, Ana Torres Soto y María Jesús Rodríguez ENTRENA

\section{Referencias bibliográficas}

Blenkinsop, S., Mccrone, T., Wade, P., Y Morris, M. (2006). How do young people make choices at 14 and 16? London: Department for Education and Skills.

Bolívar, A. Y Gijón, J. (2008). Historias de vida que deshacen profecías de fracaso. Cuadernos de Pedagogía, 382, 56-59.

Boylan, R. L. Y Renzulli, L. (2017). Routes and reasons out, paths back: the influence of push and pull reasons for leaving school on students' school reengagement. Youth and Society, 49(1), 46-71. DOI: 10.1177/0044118X14522078.

Caine, V. (2013). Literature review. En D. J. Clandinin, P. Steeves y V. Caine (Eds.), Composing lives in transition: a narrative inquiry into the experiences of early school leavers (pp.15-42). Bingley: Emerald.

Campbell, C. (2015). High school dropouts after they exit school: challenges and directions for sociological research. Sociology Compass, 9(7), 619-629.

Creswell., J. W. (2013). Qualitative inquiry and research design: choosing among five approaches, 3rd edition. Thousand Oaks, CA: Sage.

Eichhorst, W. Y Rinne, U. (2016). Promoting youth employment in Europe: evidencebased policy lessons. IZA Policy Paper No. 119. Recuperado de http://ftp.iza.org/ pp119. pdf

Fredrichs, J. (2014). Eight myths of student disengagement: creating classrooms of deep learning. Thousand Oaks, CA: Corwin.

Furlong, A. (2013). Youth studies: an introduction. London: Routledge.

George, A. L., y Bennett, A. (2005). Case studies and theory development in the social sciences. Cambridge, MA: MIT Press.

Hernández de la Torre, E. (2013). El diálogo con las historias de vida: la narrativa de los incidentes críticos en la trayectoria vital de jóvenes en situación de riesgo. Revista de Educación Inclusiva, 6(1) 90-106.

Higgins, J., Vaughan, K., Phillips, H., y Dalziel, P. (2008). Education employment linkages: international literature review. Lincoln, NE: AERU Research Unit, Lincoln

University.

HoekelL, K. (2014). Youth labour markets in the early twenty-first century. En A. Mann, J. Stanley y L. Archer (Eds.), Understanding employer engagement in education: theories and evidence (pp. 66-76). New York, NY: Routledge.

International Labour Office (2016). World employment and social outlook 2016: trends for youth. Geneva: ILO.

INEE, Instituto Nacional de Evaluación Educativa (2014). La EPA y el indicador educativo "Abandono temprano de la educación y la formación". Blog del INEE, 19 de febrero de 2014. Recuperado de http://blog.educalab.es/inee/2014/02/19/laepa-y-el-indicador-educativo-abandono-temprano-de-la-educacion-y-la-formacion/

Martín García, A. V. (1995). Fundamentaron teórica y uso de las historias y relatos de vida como técnicas de investigación en pedagogía social. Aula, 7, 41-16.

Merriam, S. B., Y Tisdell, E. J. (2016). Qualitative research: a guide to design and implementation, 4th edition. San Francisco, CA: Jossey-Bass.

Mills, M., Mcgregor, G., Y Te Riele, K. (2015). 'Schools are for us': the importance of dis- 
Del abandono educativo temprano al reenganche formativo: un estudio narrativo con

biogramas

José Miguel Nieto Cano, Antonio portela pruaño, Ana Torres Soto y María Jesús Rodríguez

ENTRENA

tribution, recognition and representation to creating socially just schools. In K. Trimmer, A. L. Black y S. Riddle (Eds.), Mainstreams, margins and the spaces inbetween: new possibilities for education research (pp. 150-167). London: Routledge.

Morgan, D. L. (2008). Emergent design. En L. Given (Ed.), The Sage encyclopedia of qualitative research methods, vol. 1 (pp. 245-248). Thousand Oaks, CA: Sage.

OECD (2014). OECD Employment outlook 2014. Paris: OECD Publishing.

O'Higgins, N. (2012). This time it's different? Youth labour markets during the great recession. Comparative Economic Studies, 54, 395-412.

O'Higgins, N. (2017). Young unemployment. En A. Furlong (Ed.), Routledge handbook of youth and young adulthood, 2nd edition (pp. 141-155). London: Routledge.

Patton, M. Q. (2015). Qualitative research \& evaluation methods: integrating theory and practice, 4th edition. Thousand Oaks, CA: Sage.

Portela, A., Nieto, J. M. y Toro, M. (2009). Historias de vida: Perspectivas y experiencia sobre exclusión e inclusión escolar. Profesorado, Revista de Currículum y Formación de Profesorado, 13(3), 193-218. Recuperado de http://www.ugr.es/ recfpro/

rev133ART8.pdf

Rubin, H. J., Y Rubin, I. S. (2012). Qualitative interviewing: the art of hearing data, 3rd edition. Thousand Oaks, CA. Sage.

Rumberger, R. W. (2011). Dropping out: why students drop out of high school and what can be done about it. Cambridge, MA: Harvard University Press.

Smyth, J., y Robinson, J. (2015). 'Give me air not shelter': critical tales of a policy case of student re-engagement from beyond school. Journal of Education Policy, 30(2), 220-236.

Scarpetta, S., Sonnet, A. y Manfredi, T. (2010). Rising youth unemployment during the crisis: How to prevent negative long-term consequences on a Generation? OECD Social, Employment and Migration Working Papers, No. 106. Paris: OECD Publishing. http://dx.doi.org/10.1787/5kmh79zb2mmv-en

Silverman, D. (2014). Interpreting qualitative data, 5th edition. London: Sage.

Suárez-Ortega, M. (2012). Performance, reflexivity, and learning through biographicalnarrative research. Qualitative Inquiry, 19(3), 189-200.

Tarabinl, A. (2015). Naming and blaming early school leavers: an analysis of education policies, discourses and practices in Spain. En A. Kupfer (Ed.), Power and education: contexts of oppression and opportunity (pp. 146-166). Basingstoke: Palgrave Macmillan.

Thomas, G., Y Myers, K. (2015). The anatomy of the case study. London: Sage.

Woessman, L. (2016). The economic case for education. Education Economics, 24, 1, 3-32. doi: 10.1080/09645292.2015.1059801

Yates, S., Harris, A., Sabates, R., Y Staff, J. (2011). Early occupational aspirations and fractured transitions: a study of entry into 'NEET' status in the UK. Journal of Social Policy, 40(3), 513-534.

Yin, R. K. (2014). Case study research: design and methods, 5th edition. Thousand Oaks, CA: Sage. 
